\title{
The criterion of the existence or inexistence of transverse shock wave at wedge supported oblique detonation wave
}

\author{
Ai-Feng Wang • Wei Zhao · Zong-Lin Jiang
}

Received: 22 March 2010 / Revised: 23 July 2010 / Accepted: 27 September 2010

(C)The Chinese Society of Theoretical and Applied Mechanics and Springer-Verlag Berlin Heidelberg 2011

\begin{abstract}
A simplified theoretic method and numerical simulations were carried out to investigate the characterization of propagation of transverse shock wave at wedge supported oblique detonation wave. After solution validation, a criterion which is associated with the ratio $\Phi\left(u_{2} / u_{\mathrm{CJ}}\right)$ of existence or inexistence of the transverse shock wave at the region of the primary triple was deduced systematically by 38 cases. It is observed that for abrupt oblique shock wave (OSW)/oblique detonation wave (ODW) transition, a transverse shock wave is generated at the region of the primary triple when $\Phi<1$, however, such a transverse shock wave does not take place for the smooth OSW/ODW transition when $\Phi>1$. The parameter $\Phi$ can be expressed as the Mach number behind the ODW front for stable CJ detonation. When $0.9<\Phi<1.0$, the reflected shock wave can pass across the contact discontinuity and interact with transverse waves which are originating from the ODW front. When $0.8<\Phi<0.9$, the reflected shock wave can not pass across the contact discontinuity and only reflects at the contact discontinuity. The condition $(0.8<\Phi<0.9)$ agrees well with the ratio $\left(D_{\text {ave }} / D_{\mathrm{CJ}}\right)$ in the critical detonation.
\end{abstract}

Keywords Oblique detonation wave - Transverse shock wave Oblique shock wave Transition $\cdot$ Numerical simulations

\section{Introduction}

The supersonic flow of premixed combustible gases around a wedge generates an oblique shock wave, which can trigger

\footnotetext{
A.-F. Wang (凶) $\cdot$ W. Zhao $\cdot$ Z.-L. Jiang

Key Laboratory of High Temperature Gas Dynamics (LHD), Institute of Mechanics, Chinese Academy of Sciences, 100190 Beijing, China

e-mail: wangaifeng@imech.ac.cn
}

combustion and stabilize the combustion process eventually forming a standing oblique detonation wave. The supersonic combustion mechanism indicates the characterization of short combustion time and high combustion efficiency [1], so the standing oblique detonation wave is considered to be a promising propulsion system of hypersonic vehicles. It has been investigated both experimentally [2-5] and numerically [6-12].

Previous studies have indicated that the existence of a standing ODW is becoming possible. Nevertheless, some appropriate conditions [13] are required: (1) the velocity of the inlet flow must be larger than the detonation velocity of the Chapman-Jouguet (CJ) $D_{\mathrm{CJ}}$ and (2) the angle of the wedge must be between the angle of the Chapman-Jouguet $\theta_{\mathrm{CJ}}$ and the maximum attached angle of the ODW $\theta_{\max }$. The structure of the ODW in numerical simulations $[6-9,11]$ consists of an oblique shock wave, an induction region, a set of deflagration waves, contact discontinuity and an oblique detonation wave. The schematic diagram of the OSW/ODW structure is shown in Fig. 1. Such detonation structure has been confirmed by experimental observations $[3,4]$. But the difference between the experiments and the computations is the structure of the OSW/ODW transition region which is presented in Fig. 2. One possibility is that the wedge in the experiments is a gaseous wedge, and the wedge in the computations is a solid wedge. There are two types of transitions: one is an abrupt transition, the other is a smooth transition. Two types of transitions involve different structures of wave series. Under certain conditions such as high initial pressure [10], high wedge angle and high activation energy [12], the OSW/ODW transition becomes abrupt; a transverse shock wave appears between the wedge surface and the primary triple point, which is generated among OSW, ODW and deflagration waves, reflects from the wedge wall, passes across the contact discontinuity (shear layer) and interacts with transverse waves which originate from the ODW front. Such interaction makes the ODW cell-like structure 
irregular and complex. However, all the transverse waves propagate in the same direction behind the ODW front. Such propagation is distinct from the propagation in the two-way direction behind the normal detonation wave (NDW) front.

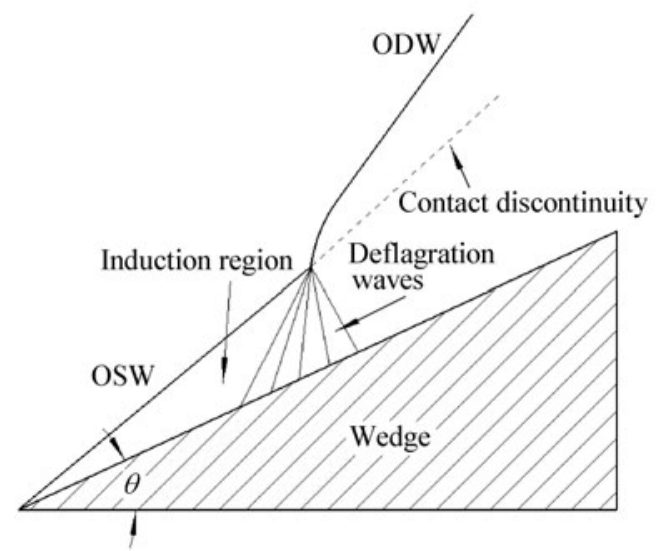

Fig. 1 Schematic diagram of OSW/ODW structure

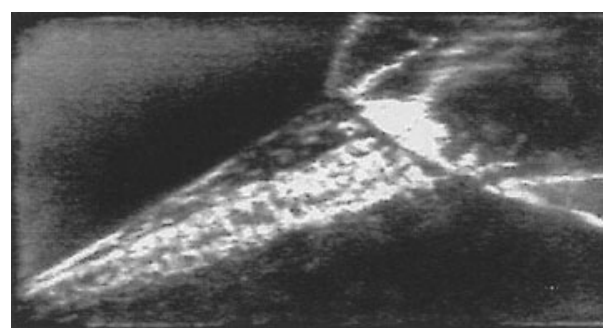

Fig. 2 Schlieren image of the OSW/ODW transition

The main purpose of this paper is to investigate the difference between two types of OSW/ODW transition and transverse wave propagation mechanisms behind the standing ODW front. As there are two types of transitions, there must be two kinds of propagation of wave series in the primary triple region. The propagation of transverse shock waves in the ODW structure is very important because of its influence on the stability of the ODW front. Accordingly, a criterion of the existence or inexistence of transverse shock waves is indispensable.

\section{Mathematical formulation and chemical kinetic model}

Assuming that the effect of viscosity and transport phenomenon is negligible, two-dimensional compressible Euler equations coupled with two-step chemical reaction equations are applied to simulate the oblique shock wave and oblique detonation wave phenomenon over a planar wedge. The governing equations in a two-dimensional Cartesian coordinate system can be summarized in the following conservation form

$\frac{\partial \boldsymbol{U}}{\partial t}+\frac{\partial \boldsymbol{F}}{\partial x}+\frac{\partial \boldsymbol{G}}{\partial y}=\boldsymbol{S}$,

$$
\begin{array}{ll}
\boldsymbol{U}=\left[\begin{array}{c}
\rho \\
\rho u \\
\rho v \\
E \\
\rho \alpha \\
\rho \beta
\end{array}\right], & \boldsymbol{F}=\left[\begin{array}{c}
\rho u \\
\rho u^{2}+p \\
\rho u v \\
(E+p) u \\
\rho u \alpha \\
\rho u \beta
\end{array}\right], \\
\boldsymbol{G}=\left[\begin{array}{c}
\rho v \\
\rho u v \\
\rho v^{2}+p \\
(E+p) v \\
\rho v \alpha \\
\rho v \beta
\end{array}\right], \quad \boldsymbol{S}=\left[\begin{array}{c}
0 \\
0 \\
0 \\
0 \\
\rho \dot{\omega}_{\alpha} \\
\rho \dot{\omega}_{\beta}
\end{array}\right],
\end{array}
$$

where $\rho, p, u, v$ are the density, the gas pressure, the velocity vector components in the directions $x$ and $y$, respectively. $E$ is the total energy per unit volume and is defined as

$E=\rho h-p+\frac{1}{2} \rho\left(u^{2}+v^{2}\right)$,

where the variable $h$ is the specific enthalpy per unit mass, $\alpha$ and $\beta$ denote the degree of chemical induction and heat release reaction of an improved two-step kinetics model proposed by Sichel [14] et al. In the model, the change of chemical components during reactions is included in the calculation of thermodynamic parameters and the reaction rates of the two-step chemical processes are expressed by

$$
\begin{aligned}
\dot{\omega}_{\alpha}= & \frac{p}{3 R_{0} T} \exp \left[35.1715-\frac{8530.6}{T}\right. \\
& \left.-7.22 \times 10^{-11} p^{2} \exp \left(\frac{21205}{T}\right)\right], \\
\dot{\omega}_{\beta}= & a(1-\beta) \exp \left(-\frac{b}{T}\right)+c,
\end{aligned}
$$

where $a=1.2 \times 10^{8}, b=8.0 \times 10^{3}, c=0$ and the unit of pressure $p$ is atm. For chemical reactions, all species are assumed to be thermally perfect gases; the specific heats at constant pressure $c_{\mathrm{p} i}$ and the specific enthalpies $h_{\mathrm{i}}$ for each species are calculated with the fitting functions expressed as Ref. [15]. Eight species that are $\mathrm{H}_{2}, \mathrm{O}_{2}, \mathrm{O}, \mathrm{H}, \mathrm{OH}, \mathrm{HO}_{2}$, $\mathrm{H}_{2} \mathrm{O}_{2}$ and $\mathrm{H}_{2} \mathrm{O}$ are selected in the paper.

$$
\begin{aligned}
\frac{C_{\mathrm{p} i}}{R_{i}}= & a_{1 i} \frac{1}{T^{2}}+a_{2 i} \frac{1}{T}+a_{3 i}+a_{4 i} T+a_{5 i} T^{2} \\
& +a_{6 i} T^{3}+a_{7 i} T^{4}, \\
\frac{h_{i}}{R_{i} T}= & -a_{1 i} \frac{1}{T^{2}}+a_{2 i} \frac{1}{T} \ln T+a_{3 i}+\frac{a_{4 i}}{2} T \\
& +\frac{a_{5 i}}{3} T^{2}+\frac{a_{6 i}}{4} T^{3}+\frac{a_{7 i}}{5} T^{4}+\frac{b_{1 i}}{T} .
\end{aligned}
$$




\section{Numerical method of solution procedure}

In order to capture the shock wave and detonation wave accurately, the fifth order weighted essentially non-oscillatory schemes (WENO) [16] are adopted to reconstruct the convection terms, and the semi-discrete finite difference equations are written as

$$
\begin{aligned}
\frac{\partial \tilde{\boldsymbol{U}}_{i, j}}{\partial t}= & -\frac{1}{\Delta x}\left(\overline{\boldsymbol{F}}_{i+\frac{1}{2}, j}-\overline{\boldsymbol{F}}_{i-\frac{1}{2}, j}\right) \\
& -\frac{1}{\Delta y}\left(\overline{\boldsymbol{G}}_{i, j+\frac{1}{2}}-\overline{\boldsymbol{G}}_{i, j-\frac{1}{2}}\right)+\tilde{S}_{i, j},
\end{aligned}
$$

where

$$
\begin{aligned}
& \overline{\boldsymbol{F}}_{i+\frac{1}{2}, j}=\tilde{\boldsymbol{F}}_{i+\frac{1}{2}, j}^{+}+\tilde{\boldsymbol{F}}_{i+\frac{1}{2}, j}^{-}, \\
& \overline{\boldsymbol{G}}_{i+\frac{1}{2}, j}=\tilde{\boldsymbol{G}}_{i, j+\frac{1}{2}}^{+}+\tilde{\boldsymbol{G}}_{i, j+\frac{1}{2}}^{-},
\end{aligned}
$$

where $\tilde{\boldsymbol{F}}^{ \pm}$and $\tilde{\boldsymbol{G}}^{ \pm}$stand for the flux vector splitting according to Steger-Warming method [17], which is employed to account for upwind effect. The time integration is completed with the third order TVD Runge-Kutta method.

The computational domain is presented in Fig. 3. In the numerical experiments the stoichiometric hydrogen-oxygen mixture enters the computational domain, which is a rectangular area surrounded by a double dot dash line. The size of the rectangular area is $0.01 \mathrm{~m} \times 0.005 \mathrm{~m}$, and initial pressure $p_{0}$ and temperature $T_{0}$ are $40 \mathrm{kPa}$ and $300 \mathrm{~K}$. Since two types of OSW/ODW transitions are required, the wedge angle and Mach number of the inlet flow should be suitable for two types in order to analyze different wave propagation. In this paper, grid resolution $(1005 \times 505)$ with grid size $\Delta x=\Delta y=0.01 \mathrm{~mm}$ is considered. Five ghost points are added to the outside of the boundary in order to accept the flow flux at the boundary according to five order WENO scheme.

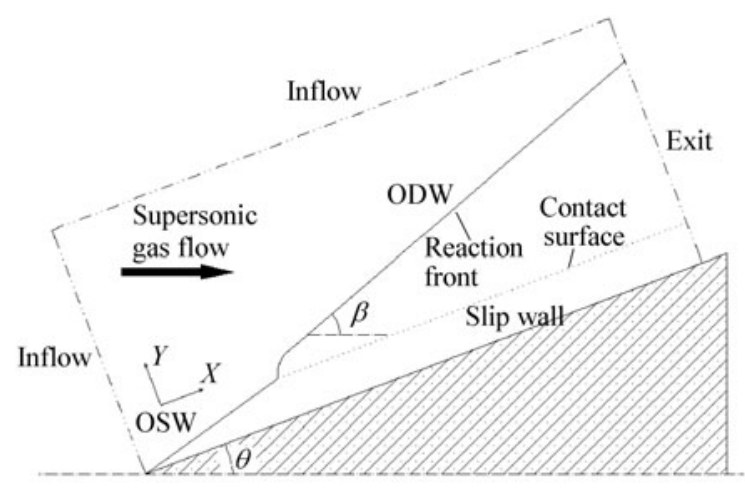

Fig. 3 Schematic diagram of OSW/ODW computational domain

Inlet flow boundary conditions are fixed at the initial value, and at the exit the flow parameters are extrapolated from the interior. Since the solid surface of the wedge is assumed to neglect viscosity, slip boundary conditions are used $(\partial u / \partial n=v=0)$ where $n$ is the local normal to the surface.

\section{Validation of numerical methods}

In order to explore propagation mechanics of the detonation wave with numerical CFD technology, the validation of numerical methods is necessary to demonstrate the reliability of numerical results. Two cases are chosen. The first case is a planar CJ detonation propagation in a straight tube with a length of $0.1 \mathrm{~m}$ to check the detonation model. The second is the two-dimensional oblique detonation wave to check the structure of the ODW.

In the first case, the planar detonation propagation was simulated at initial conditions of $p_{0}=100 \mathrm{kPa}$ and $T_{0}=293.0 \mathrm{~K}$ with $\Delta x=0.05 \mathrm{~mm}$. The predicted pressure distributions at several instants were plotted in Fig. 4. The pressure spikes experience sharp variations at the beginning of the propagation due to the ignition effect. The statistics of the numerical data showed that the detonation speed is $2839.64 \mathrm{~m} / \mathrm{s}$, and the pressure and temperature at the $\mathrm{CJ}$ point according to the condition $\left(D_{\mathrm{CJ}}=u_{\mathrm{CJ}}+a_{\mathrm{CJ}}\right)$ are $1.841 \mathrm{MPa}$ and $3651.5 \mathrm{~K}$. The corresponding data calculated with the detailed chemical reaction mechanism are $2845.41 \mathrm{~m} / \mathrm{s}$ for the detonation speed, $1.845 \mathrm{MPa}$ for the CJ pressure and $3673.6 \mathrm{~K}$ for the $\mathrm{CJ}$ temperature, respectively. The maximum discrepancy is about $0.60 \%$. Hence, the reliability of the present chemical reaction model was well demonstrated.

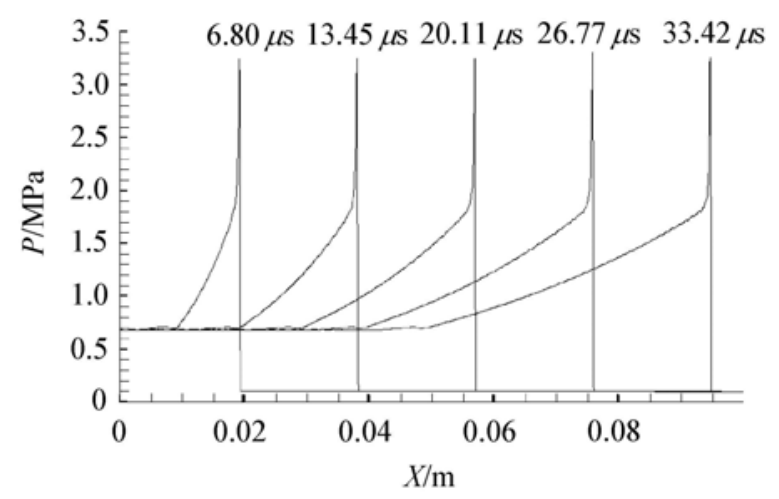

Fig. 4 Pressure distributions at different instants showing planar detonation propagation

In the second case, the inlet stoichiometric mixture with the inlet Mach number 7.5 over a $25^{\circ}$ wedge generates OSW/ODW structures and an abrupt OSW/ODW transition. The numerical result and an experimental schlieren image are presented together in Fig. 5. The comparison of these two figures shows that the whole structure of flow looks roughly the same both in the experimental and numerical results. The main structure consists of OSW, ODW, triple point and contact surface. Therefore, the reliability of the simulated solution of the structure of the OSW/ODW was also demonstrated. 


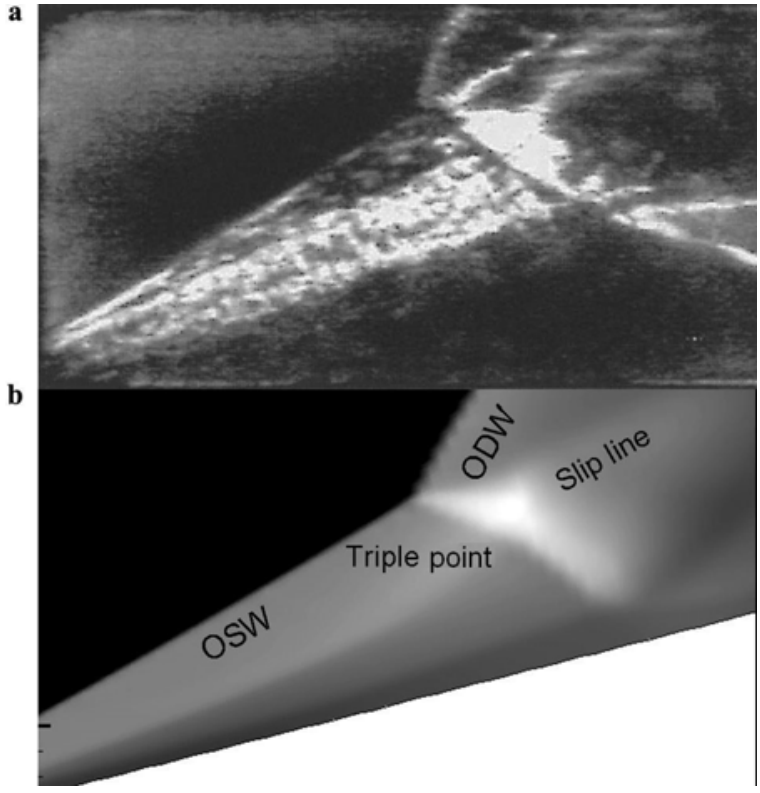

Fig. 5 OSW/ODW structure over a $25^{\circ}$ wedge with a mixture of stoichiometric $\mathrm{H}_{2}$-Air at $p_{0}=40 \mathrm{kPa}, T_{0}=293.0 \mathrm{~K}, M a=7.5$. a Experimental schlieren image; b Numerical density contour

\section{Results and discussion}

5.1 Transverse shock wave at two types of OSW/ODW transition

Three different inlet Mach numbers have been used for the OSW/ODW transition simulation. The pressure and temperature fields have been presented in Fig. 6. Previous studies show that the type of transition obtained numerically is influenced by the ratio between the heat release time, $t_{\mathrm{q}}$, and the induction time of the chemical kinetics, $t_{\mathrm{i}, \text {. }}$ When $t_{\mathrm{q}} / t_{\mathrm{i}}$ is large, smooth transitions are more likely to be obtained (see Figs. 6b, and 6c) and, on the contrary, when $t_{\mathrm{q}} / t_{\mathrm{i}} \rightarrow 0$, abrupt transitions are found to occur (see Fig. 6a). When the Mach number changes from 7.5 to 8.5 , the increase of pressure and temperature behind the oblique shock wave reduces the induction time of chemical kinetics $t_{\mathrm{i}}$ and elevates the ratio of $t_{\mathrm{q}} / t_{\mathrm{i}}$ so as to make the transitions smoother. A special phenomenon takes place at the region of the primary triple, a transverse shock wave is generated in an abrupt transition in Fig. 6a and the transverse shock wave also takes place in the experiment (Fig. 2). However, the transverse shock wave is very weak or can not stand in the smooth transition. Although the previous work can explain the level of the transition, the existence or inexistence of the transverse shock wave in the region of primary triple needs a criterion. Accordingly the following investigations are about the difference between the two types of transitions and the criterion of the existence or inexistence of the transverse shock wave.
Pressure distributions at the region of the primary triple with three inlet Mach numbers $(7.5,8.0$, and 8.5) is shown in Fig.7. The pressure profile indicates a sharper pressure spike for $M a=7.5$ than for $M a=8.0,8.5$. The pressure distributions are relatively smooth in the downstream region for $M a=8.0,8.5$, but become oscillatory for $M a=7.5$. This phenomenon may be attributed to the disturbance of the transverse shock wave at the primary triple. Figure 8 shows Mach number distributions for three initial Mach numbers at the region of the primary triple. When the initial Mach number is 7.5 , the region of the primary triple is subsonic and the minimum local Mach number is about 0.6. However, the overall flow field is supersonic for $M a=8.0,8.5$. Therefore there are two different OSW/ODW propagation mechanics at different Mach numbers, and moreover, the transverse shock wave at the region of the primary triple does not exist in all cases, the existence or inexistence of the transverse shock wave seems associated with the local Mach number.

In order to find a criterion of the existence or inexistence of the transverse shock wave, a schematic diagram of a simplified planar ODW model is shown in Fig. 9. There are three assumptions: (1) the incoming flow is premixed combustible gases which are mixed uniformly and moving stability; (2) the chemical reaction layer is equal to heat reaction; (3) the flow is considered to be inviscid and adiabatic. For a given wedge angle, equations for mass momentum, and energy in the direction normal to the ODW are calculated as follows

$$
\begin{aligned}
& \rho_{1} u_{1 n}=\rho_{2} u_{2 n}, \\
& P_{1}+\rho_{1} u_{1 n}^{2}=P_{2}+\rho_{2} u_{2 n}^{2}, \\
& C_{p 1} T_{1}+Q+\frac{u_{1 n}^{2}}{2}=C_{p 2} T_{2}+\frac{u_{2 n}^{2}}{2} .
\end{aligned}
$$

The subscript " $n$ " refers to flow normal to the ODW. With some special manipulations, the final equation can be expressed as

$$
\begin{aligned}
& \frac{(2 A-1) \gamma_{1}+1}{2} M_{1 n}^{2} X^{2}-A\left(1+\gamma_{1} M_{1 n}^{2}\right) X \\
& \quad+\left(1+\frac{\gamma_{1}-1}{2} M_{1 n}^{2}\right)+\tilde{Q}=0 \\
& A=\frac{\gamma_{2}\left(\gamma_{1}-1\right)}{\gamma_{1}\left(\gamma_{2}-1\right)} \\
& X=\frac{\tan (\beta-\theta)}{\tan \beta} \\
& M_{1 n}^{2}=M_{1}^{2} \sin ^{2} \beta \\
& \tilde{Q}=Q / C_{p 1} T .
\end{aligned}
$$

The equation is called $\theta-\beta-M$ relation, and species $\theta$ as a unique function of $\beta$ and $M_{1}$. $\theta$ is the wedge angle, $\beta$ is the angle of ODW, $\tilde{Q}$ is the nondimensional heat reaction parameter, $C_{\mathrm{p} 1}$ is the specific heat at constant pressure of incoming flow. 

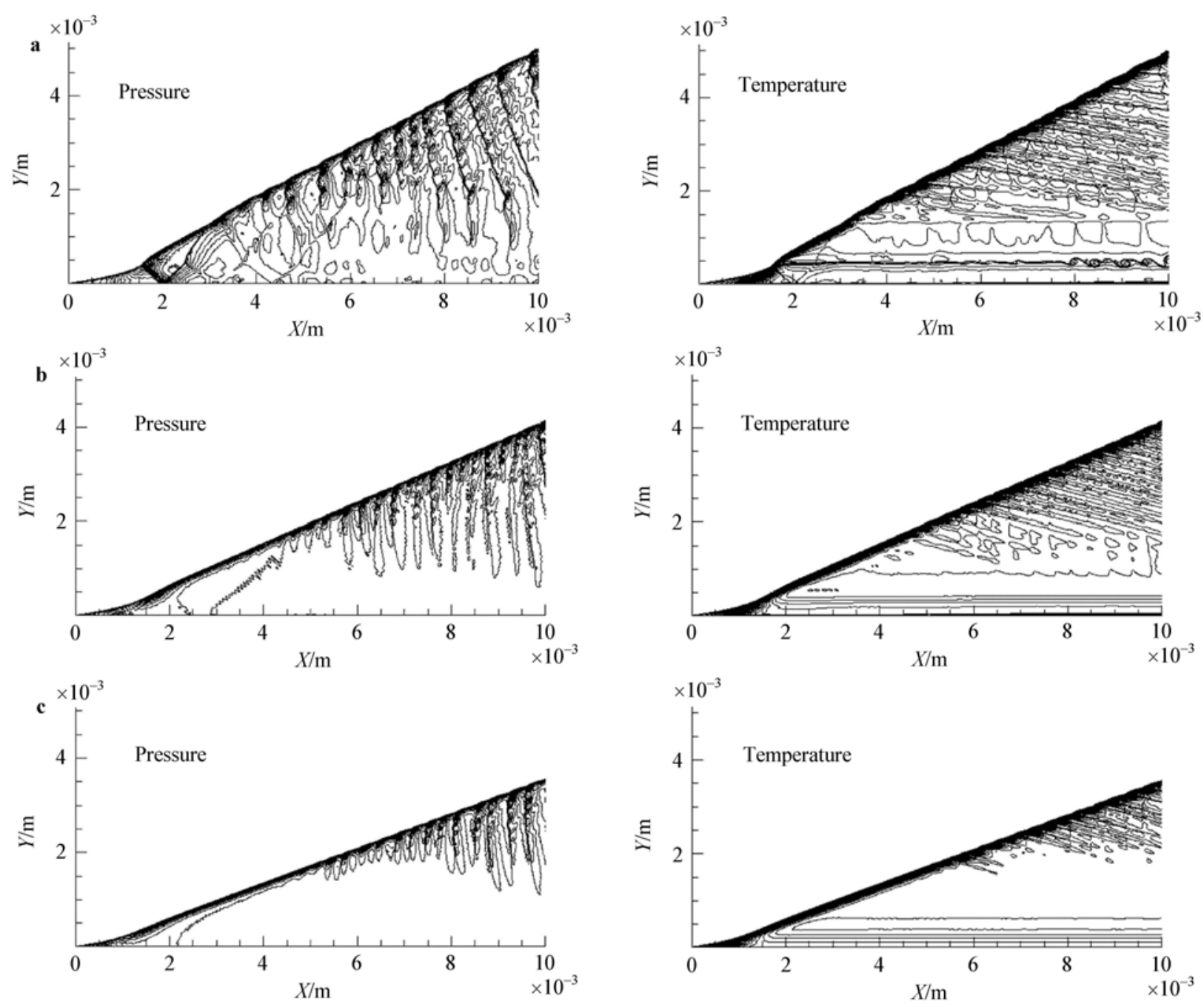

Fig. 6 Pressure and temperature fields for successive Mach numbers at $p_{0}=40 \mathrm{kPa}, T_{0}=300.0 \mathrm{~K}$ and $\theta=30^{\circ}$. a $M a=7.5$; b $M a=8.0$; c $M a=8.5$

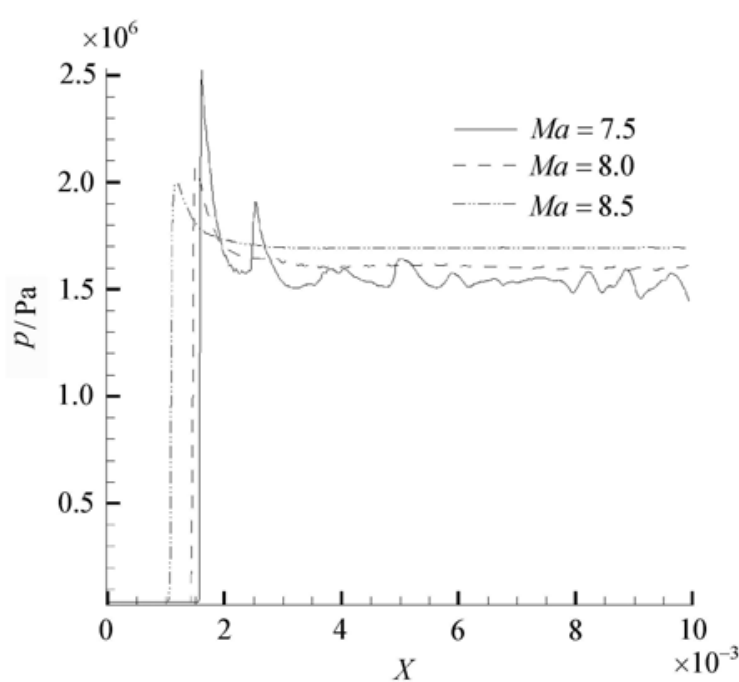

Fig. 7 Pressure distributions at the region of the primary triple with three initial Mach number

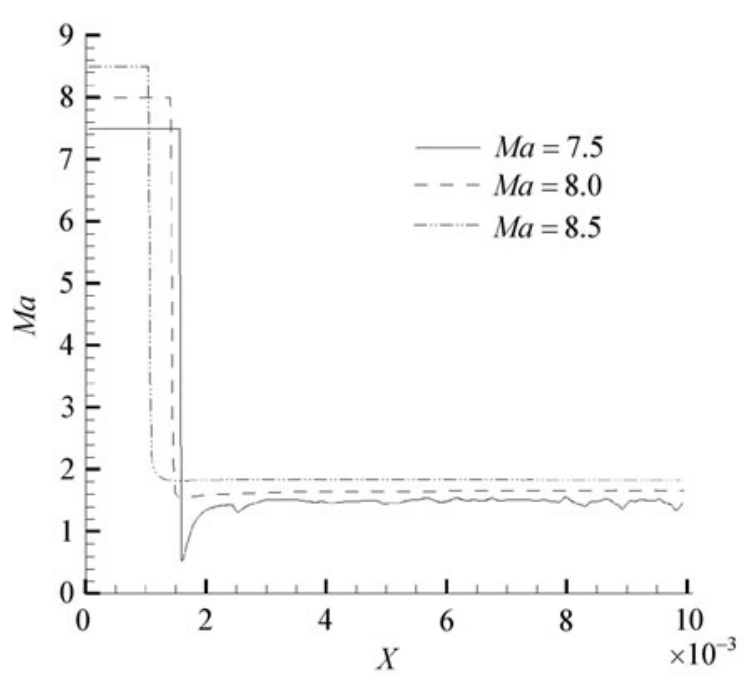

Fig. 8 Mach number distributions at the region of the primary triple with three initial Mach number 


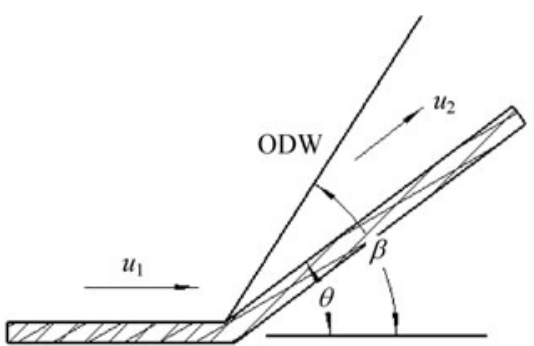

Fig. 9 Schematic diagram of simplified planar ODW model

We have, for a calorically perfect gas

$$
\begin{aligned}
& \frac{\rho_{2}}{\rho_{1}}=\frac{(\gamma+1) M_{1 n}^{2}}{(\gamma-1) M_{1 n}^{2}+2}, \\
& \frac{p_{2}}{p_{1}}=1+\frac{2 \gamma}{\gamma+1}\left(M_{1 n}^{2}-1\right), \\
& M_{2 n}^{2}=\frac{M_{1 n}^{2}+2 /(\gamma-1)}{[2 \gamma /(\gamma-1)] M_{1 n}^{2}-1} .
\end{aligned}
$$

Through the above equations, $u_{2}$ can be defined as

$u_{2}=M_{2} a_{2}=\frac{M_{2 n}}{\sin (\beta-\theta)} a_{2}=\frac{M_{2 n}}{\sin (\beta-\theta)} \sqrt{\gamma \frac{p_{2}}{\rho_{2}}}$,

where $\gamma=\frac{1}{2}\left(\gamma_{1}+\gamma_{2}\right)$.

The CJ velocity $u_{\mathrm{CJ}}$ is calculated with the detailed chemical reaction mechanism.

The nondimensional ratio $\Phi$ between $u_{2}$ and $u_{\mathrm{CJ}}$ is defined as

$\Phi=u_{2} / u_{\mathrm{CJ}}$.

In order to investigate the criterion of the existence or inexistence of the transverse wave, 38 cases $\left(p_{0}=40 \mathrm{kPa}\right.$, $\left.T_{0}=300.0 \mathrm{~K}\right)$ are simulated. In 38 cases which are satisfied with two conditions of the existence of a standing ODW, 16 cases are simulated for different initial Mach numbers and others are simulated for different wedge angles. The results of all cases are presented in Fig. 10 and Fig. 11.

Figure 10 shows $\Phi$ distributions at different initial Mach numbers. The ratio $\Phi$ strengthens with the increase of the initial Mach number. In 16 cases, there are two different phenomenons. When $\Phi<1$ (marked with triangle), the transverse shock wave takes place at the region of primary triple. When $\Phi>1$ (marked with circle), the transverse shock wave can not emerge at the region of primary triple. The wedge angle $\theta$ is also a crucial component in the studying of the ODW, so the influence of the wedge angle on the transverse shock wave can not be ignored. Figure 11 shows $\Phi$ distributions at different wedge angles. When $M a=7.5$ and for increasing $\theta, \Phi$ decreases until about $30^{\circ}$, where it begins to increase. Increasing the Mach number to 8.0 has the effect of increasing the ratio $\Phi$ and moving the minimum to a higher wedge angle. Similarly, the results of simulations of 22 cases indicate that transverse shock wave exists when $\Phi<1$ and does not exist when $\Phi>1$.

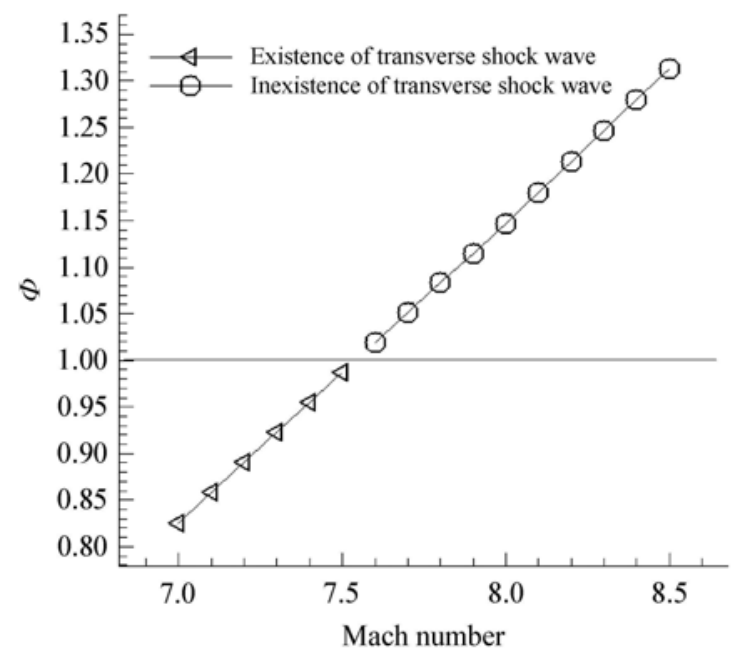

Fig. $10 \Phi$ distributions at different initial Mach number $\left(\theta=30^{\circ}\right)$

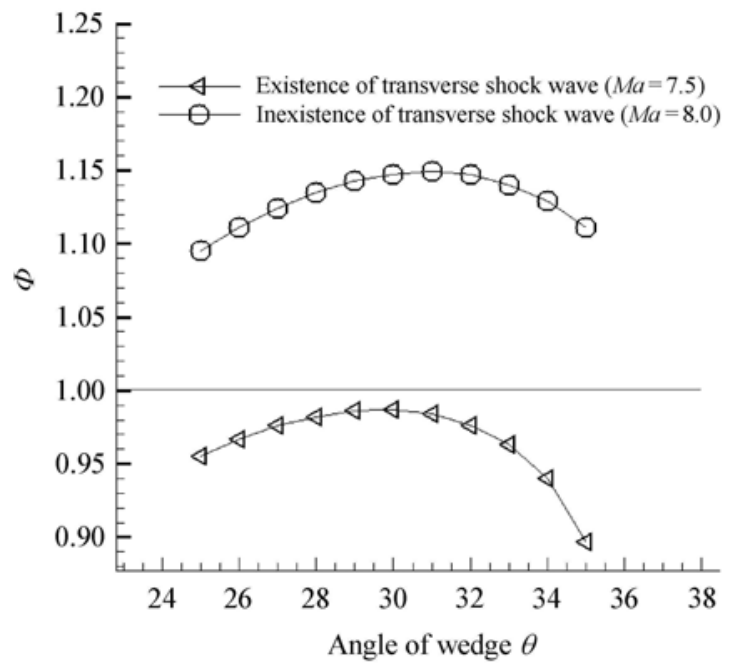

Fig. $11 \Phi$ distributions at different wedge angles

Under the above study, a criterion of the existence or inexistence of the transverse shock wave has been deduced by theoretical formula and numerical simulations. Special form is expressed as follows

Transverse shock wave $=\left\{\begin{array}{l}\text { True }(\Phi<1), \\ \text { False }(\Phi>1) .\end{array}\right.$

However, the above formula is based on two conditions of a standing ODW which are mentioned in the introduction.

\subsection{Experimental verification}

In order to verify the criterion, Broda's experimental results [18] were reported in this paper. The schlieren images of two types of OSW/ODW transition are shown in Fig. 12 and Fig. 13. Figure 12 shows the flow field where the OSW/ODW transition is abrupt. A transverse shock wave 
takes place in the flow field $\left(M a_{0}=6.7, P_{0}=30 \mathrm{kPa}\right.$, $\left.T_{0}=293 \mathrm{~K}, \mathrm{C}_{2} \mathrm{H}_{2}+2.5 \mathrm{O}_{2}+10.5 \mathrm{Ar}\right)$. Computed through Eq. (12), the value of $\Phi$ is 0.869 . The result is satisfied with the criterion (Eq. (13)). The smooth type of OSW/ODW transition is presented in Fig. 13 because there is not a transverse shock wave in the flow field $\left(M a_{0}=8.8, P_{0}=30 \mathrm{kPa}\right.$, $T_{0}=293 \mathrm{~K}, 2 \mathrm{H}_{2}+\mathrm{O}_{2}+3.76 \mathrm{~N}_{2}$ ). Calculated through Eq. (12), the value of $\Phi$ is 1.673 . The result is also in accord with the criterion. Therefore, the criterion of existence or inexistence of the transverse shock wave in the paper is reliable.

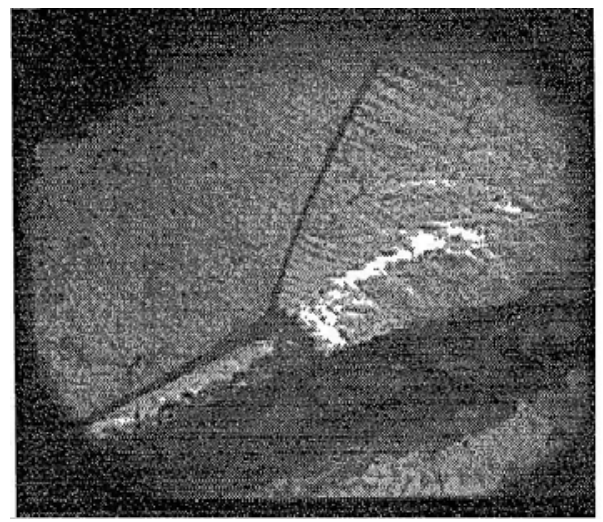

Fig. 12 Schlieren image of the OSW/ODW transition (Abrupt) $\left(M a=6.7, P_{0}=30 \mathrm{kPa}, T_{0}=293 \mathrm{~K}, \mathrm{C}_{2} \mathrm{H}_{2}+2.5 \mathrm{O}_{2}+10.5 \mathrm{Ar}\right)$

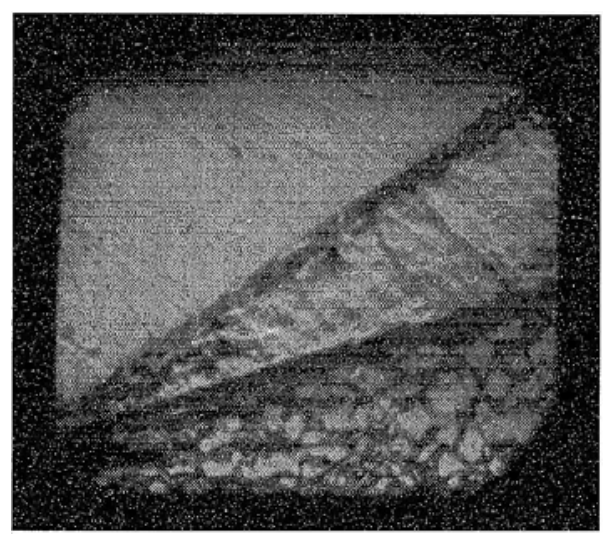

Fig. 13 Schlieren image of the OSW/ODW transition (Smooth) $\left(M a=8.8, P_{0}=30 \mathrm{kPa}, T_{0}=293 \mathrm{~K}, 2 \mathrm{H}_{2}+\mathrm{O}_{2}+3.76 \mathrm{~N}_{2}\right)$

\subsection{Physical description of $\Phi$}

In the above study, the nondimensional $\Phi$ is defined in Eq. 12. In general, for a stable CJ detonation,

$u_{\mathrm{CJ}}=a_{\mathrm{CJ}}$.

Substitute Eq. (14) into Eq. (12)

$\Phi=u_{2} / u_{\mathrm{CJ}}=u_{2} / a_{\mathrm{CJ}}=M a_{\mathrm{NT}}$.

From Eq. (15), $\Phi$ is the Mach number behind the ODW front for stable CJ detonation. However, $\Phi$ is not the traditional Mach number, subscript NT refers to non-traditional.

\subsection{Propagation of transverse shock wave at the wedge}

Figure 14 shows calculated pressure and temperature contours of OSW/ODW structure. A transverse shock wave is generated from the region of the primary triple, reflects from the wedge wall, passes across the contact discontinuity and interacts with transverse waves which originate from the ODW front. The result is consistent with the work of Choi et al. However, when $M a=7.2$ (In Fig. 15), the reflected shock wave can not pass across the contact discontinuity and reflections multiply between the wedge and the contact discontinuity. Therefore, the ratio $\Phi$ as the object of such study is listed as follows.

In Table 1, there are different propagations of transverse shock wave at different ratio $\Phi$. When $0.9<\Phi<1.0$, the reflected shock wave can pass across the contact discontinuity and form an oblique shock wave which can strengthen the ODW front. When $0.8<\Phi<0.9$, the reflected shock wave can not pass across the contact discontinuity and only reflects between the contact discontinuity and the wedge surface. The condition $(0.8<\Phi<0.9)$ agrees well with the ratio $\left(D_{\text {ave }} / D_{\mathrm{CJ}}\right)$ in the critical detonation [19] (single helical detonation where the number of transverse wave is one). Therefore, when $0.8<\Phi<0.9$, the oblique detonation is a critical detonation.

Table 1 Refraction and $\Phi$ distributions different Mach number

\begin{tabular}{lllll}
\hline Case & $M a$ & $\theta /\left(^{\circ}\right)$ & $\Phi$ & Refraction \\
\hline 1 & 7.1 & 30 & 0.858 & False \\
2 & 7.2 & 30 & 0.891 & False \\
3 & 7.3 & 30 & 0.923 & True \\
4 & 7.4 & 30 & 0.955 & True \\
5 & 7.5 & 30 & 0.987 & True \\
\hline
\end{tabular}

\section{Summary and conclusions}

Characterization of the propagation of transverse shock waves on wedge supported oblique detonation waves was examined systematically in the paper with different Mach numbers and wedge angles. It was confirmed that the transverse shock wave at the region of the primary triple exists in the abrupt OSW/ODW transition in which the Mach number is lower in our numerical study and can not stand in the smooth transition.

A criterion was deduced by theoretical formula and numerical simulations. The evolution criterion is the ratio between the local velocity and the CJ velocity. A transverse shock wave exists when $\Phi<1$ and can not exist when $\Phi>1$. The experimental results can support the criterion in the paper.

The parameter $\Phi$ can be expressed as the Mach number behind the ODW front for stable CJ detonation. 

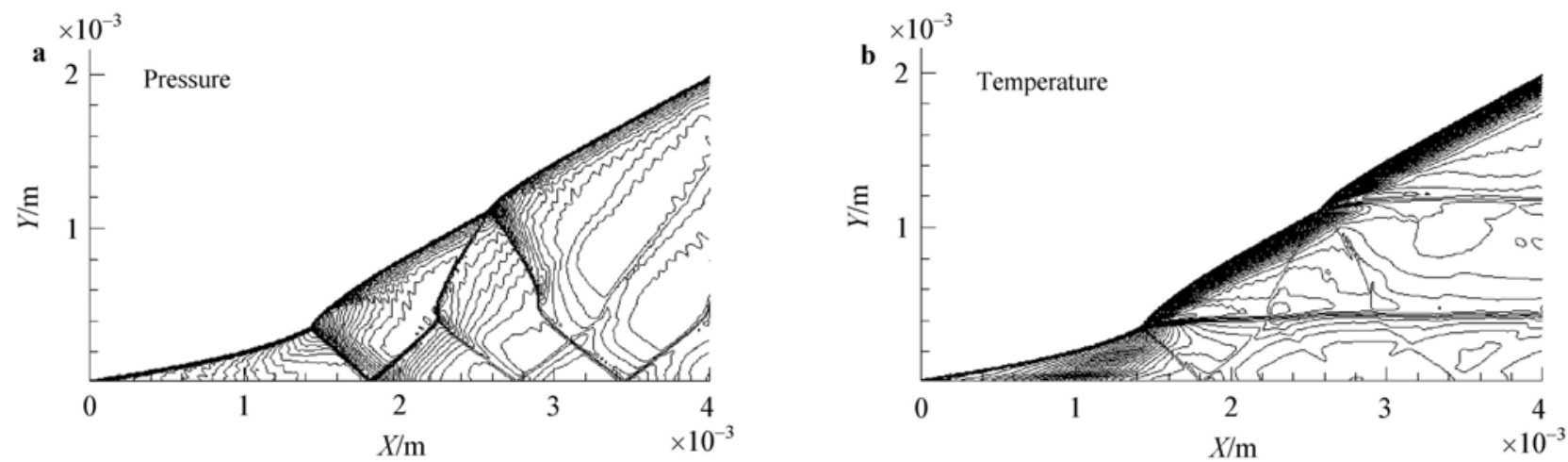

Fig. 14 Pressure and temperature contours of OSW/ODW structure at $p_{0}=40 \mathrm{kPa}, T_{0}=300.0 \mathrm{~K}, \theta=30^{\circ}$ and $M a=7.4$
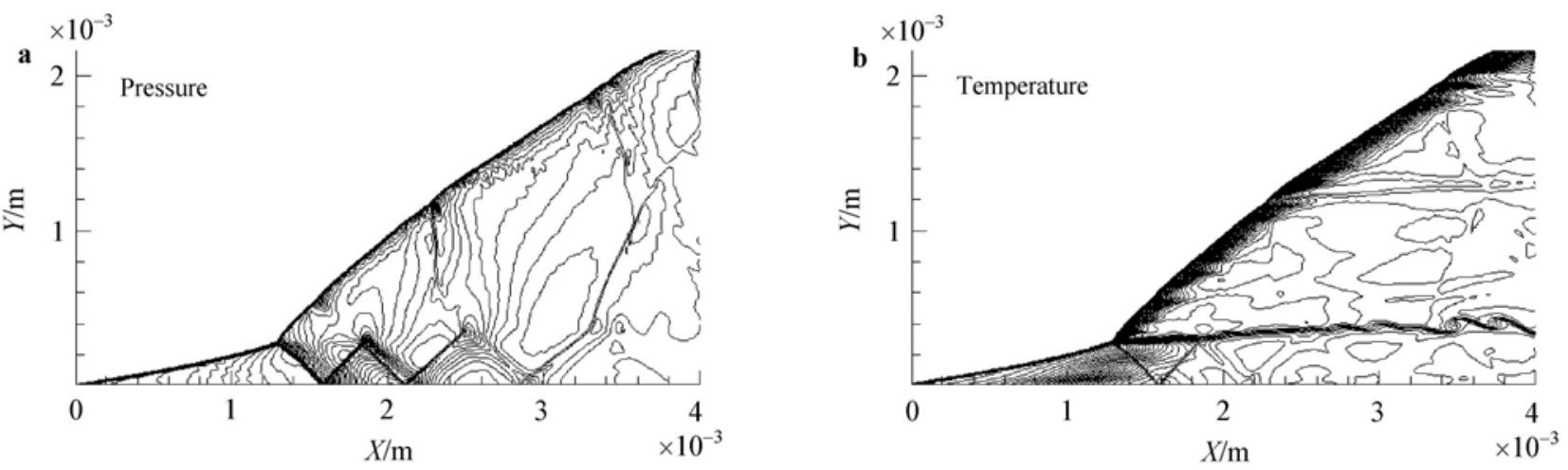

Fig. 15 Pressure and temperature contours of OSW/ODW structure at $p_{0}=40 \mathrm{kPa}, T_{0}=300.0 \mathrm{~K}, \theta=30^{\circ}$ and $M a=7.2$

In the wedge, the transverse shock wave reflected from the wedge wall, passes across the contact discontinuity at $0.9<\Phi<1.0$ and forms an oblique shock wave which can strengthen the ODW front, but the transverse shock wave which reflected multiplies between the wedge and the contact discontinuity can not pass across the contact discontinuity at $0.8<\Phi<0.9$. When $0.8<\Phi<0.9$, the oblique detonation is a critical detonation.

\section{References}

1 Smeets, G.: Shock tube investigations of $h_{2}$ combustion in a high temperature supersonic air flow (scramjet). IUTAM Symposium on Combustion in Supersonic Flows. Chamption, M., andDeshaies, B., eds. Kluwer (1995)

2 Lehr, H.F.: Experiments in shock induced combustion. Astronautica Acta 17, 589-597 (1972)

3 Dabora, E.K., Desbordes, D., Wagner, H.G.: Oblique detonation at hypersonic velocities. In: Kuhl A.L., Leyer J.C., Borisov A.A., Sirignano W.A., eds. Dynamics of Detonations and Explosions: Detonations, AIAA, 187-201 (1991)

4 Viguier, C., Gourara, A., Desbordes, D.: Onset of oblique detonation waves: comparison between experimental and numerical results for hydrogen-air mixtures. In: Proceedings of Twenty-Seventh Symposium (international) on Combustion, 3023-3031 (1996)

5 Jiro, K., Takakage, A., Shingo, C., et al.: Criticality for stabilized oblique detonation waves around spherical bodies in acetylene/oxygen/krypton mixture. In: Proceedings of the Combustion Institute 29, 2817-2824 (2002)

6 Li, C., Kailasanath, K., Oran, E.S.: Detonation structures behind oblique shocks. Physics of Fluids 6, 1600-1611 (1994)

7 Fujiwara, T., Matsuo, A.: Two-dimensional detonation supported by a blunt body or a wedge. AIAA Paper, 0088-0098 (1988)

8 Fernando, L., Figueira da Silva, L.F., Deshaies, B.: Stabilization of an oblique detonation wave by a wedge: A parametric numerical study. Combustion and Flame 121(1\&2), 152-166 (2000)

9 Miltiadis, V.P.: A numerical study of wedge-induced detonations. Combustion and Flame 120, 526-538 (2000)

10 Pimentel, C.A.R., Azevedo, J.L.F., Figueira da Silva, L.F., et al.: Numerical study of wedge supported oblique shock waveoblique detonation wave transitions. J. of the Braz. Soc. Mechanical Sciences XXIV, 149-157 (2002)

11 Teng, H., Zhao, W., Jiang, Z.: A novel oblique detonation structure and its stability. Chinese Physics Letter 24(7), 19851988(2007)

12 Choi, J.Y., Kim, D.W., Jeung, I.S., et al.: Cell-like structure of unstable oblique detonation wave from high-resolution numer- 
ical simulation. Proceedings of the Combustion Institute 31(2), 2473-2480 (2007)

13 Pratt, D.T., Humphrey, J.W.: Morphology of standing oblique detonation waves. Propulsion and Power 7(5), 837-845 (1991)

14 Sichel, M., Tonello, N., Oran, E., et al.: A two-step kinetics model for numerical simulation of explosions and detonations in $\mathrm{H}_{2}-\mathrm{O}_{2}$ mixture. Proc. R. Soc. Lond. A 458, 49-82 (2001)

15 McBride, B., Zehe, M., Gordon, S.: NASA glenn coefficients for calculating thermodynamics properties of individual species. NASA/TP 211556 (2002)

16 Shu, C.W., Osher, S.: Efficient implementation of essentially non-oscillatory shock capturing schemes II. J. Computational Physics 83, 32-78 (1989)

17 Steger, J., Warming, R.: Flux vector splitting of the invicid gasdynamics equations with applications to finite difference methods. J. Comp. Phys. 40, 263-293 (1981)

18 Broda, J.C.: An experimental study of oblique detonation waves. [Ph.D. Thesis]. USA: The University of Connecticut, 1993

19 Zhou, K.Y., Chen, C.G.: Detonation Theory and Application. Hefei: Press of University of Science and Technology of China, 1996 (in Chinese) 